\title{
Corela
}

Cognition, représentation, langage

HS-12 | 2012

Langue, espace, cognition

\section{Le centre n'est pas au milieu (et inversement). Pour une approche phénoménologique et gestaltiste de la localisation}

Philippe Gréa

\section{OpenEdition}

Journals

Édition électronique

URL : http://journals.openedition.org/corela/2831

DOI : $10.4000 /$ corela.2831

ISSN : 1638-573X

Éditeur

Cercle linguistique du Centre et de l'Ouest - CerLICO

Référence électronique

Philippe Gréa, « Le centre n'est pas au milieu (et inversement). Pour une approche phénoménologique et gestaltiste de la localisation », Corela [En ligne], HS-12 | 2012, mis en ligne le 19 décembre 2012, consulté le 10 décembre 2020. URL : http://journals.openedition.org/corela/2831 ; DOI : https:// doi.org/10.4000/corela.2831

Ce document a été généré automatiquement le 10 décembre 2020.

\section{(c) (i) (2)(2)}

Corela - cognition, représentation, langage est mis à disposition selon les termes de la licence Creative Commons Attribution - Pas d'Utilisation Commerciale - Partage dans les Mêmes Conditions 4.0 International. 


\section{Le centre n'est pas au milieu (et inversement). Pour une approche phénoménologique et gestaltiste de la localisation}

Philippe Gréa

\section{NOTE DE L'AUTEUR}

Cet article a bénéficié des discussions que nous avons eues avec Florence Villoing et Richard Huyghe que nous remercions au passage. Toutes les erreurs qui subsisteraient sont de notre entière responsabilité.

\section{Introduction : les limites d'une caractérisation purement spatiale de centre et milieu}

1 Les noms centre et milieu appartiennent, dans la terminologie de (Borillo, 1999), à la classe des «Noms de Localisation Interne» (NLI). Leur particularité tient dans le fait de combiner localisation spatiale et partition. Ils permettent en effet de désigner la partie d'un tout, et en même temps qu'elle est constituée, cette partie se trouve localisée par rapport au tout. Le centre ou le milieu d'une table désigne un morceau de la table, mais aussi un morceau qui se situe à une distance à peu près identique des différents bords de la table.

2 En combinaison avec la préposition à, centre et milieu construisent des locutions prépositionnelles locatives, au milieu de et au centre de. Une expression de la forme $\left[\mathrm{NP}_{0}\right.$ au \{centre / milieu\} de $\mathrm{NP}_{1}$ ] permet alors d'établir une relation entre deux entités distinctes, l'entité à localiser $\left(\mathrm{NP}_{0}\right)$ et l'entité servant de système de repère $\left(\mathrm{NP}_{1}\right)$. Dans (Gréa, 2005), 
nous avons avancé une hypothèse qui permettait de distinguer les deux locutions : le caractère délimité (ou non) de $\mathrm{NP}_{1}$.

3 Depuis, un point de vue qui nous a été opposé s'appuie sur une représentation géométrique du sens de centre et de milieu et ne reconnaît pas la délimitation du $\mathrm{NP}_{1}$ comme critère pertinent. Selon cette approche, au centre de imposerait deux contraintes : (i) $\mathrm{NP}_{1}$ dénoterait une entité au moins bidimensionnelle, et (ii) à l'image d'un cercle et de son centre, $\mathrm{NP}_{0}$ désignerait un point ou une région située à équidistance des limites de l'entité désignée par $\mathrm{NP}_{1}$. Le milieu, quant à lui, désignerait de façon moins précise, comme le TLF le suggère, « un endroit relativement éloigné des bords, de la périphérie ». Cette approche a l'avantage de s'appuyer sur un concept géométrique, l'équidistance, qui semble facilement vérifiable dans les faits.

Le problème, c'est que beaucoup d'exemples demeurent hors de portée de cette explication, voire, la contredisent. En premier lieu, le postulat (i) selon lequel $\mathrm{NP}_{1}$, dans au centre de $N P_{1}$, doit être (au moins) bidimensionnel est sérieusement remis en cause par les exemples suivants :

(1) a. Le coup d'envoi est donné au centre de la ligne médiane. (internet)

b. Le wagon restaurant est situé au centre du train. (internet, brochure artesia)

c. Balzac est au centre de la rangée. / Il faut placer le jeton au centre de la rangée horizontale.

d. On place une tomate cerise au centre de la brochette et des morceaux de poivron à chaque extrémité. (internet, recette de la brochette de poulet marinade)

e. Le pendentif est monté sur la grosse perle de rocaille bleue située au centre du collier. (internet, collier « ténèbres »)

Ces énoncés présentent tous un $\mathrm{NP}_{1}$ unidimensionnel ou conçu comme tel. Dans le dernier exemple, insistons sur le fait que la perle n'est pas au centre d'un cercle qui serait formé par le collier : elle est au contraire sur le collier, à égale distance des deux parties du fermoir, ce qui montre bien que l'intuition première tendant à privilégier l'idée d'un cercle ne tient pas.

$6 \mathrm{Si}$, pour des raisons surtout extra-linguistiques (mathématiques), les puristes répugneront à parler du centre d'une droite, le centre d'un segment ne soulève quant à lui aucune objection («le point $\mathrm{C}$ au centre du segment $\mathrm{AB} »)$. Table constitue un autre cas intéressant, car si au centre de la table peut désigner la région située à égale distance des bords de la table (bidimensionnelle), comme dans ce qui suit,

(2) Au centre de la table fumait déjà la soupière.

il existe aussi un autre emploi, tout aussi fréquent, où table correspond au contraire à une entité conçue comme unidimensionnelle :

(3) a. Christ, assis, lui treizième, au centre de la table.

b. Au centre de la table est un ancien ambassadeur, sénateur aujourd'hui.

7 On conviendra que dans ces contextes le Christ ou l'ambassadeur ne sont pas posés sur la table comme la soupière en 2. Ils sont au contraire positionnés sur l'un des côtés de la table (généralement, le plus long) ou encore sur le cercle formé par les commensaux (et non à l'intérieur de ce cercle), de façon analogue au pendentif de l'exemple 1e, c'est-àdire sur un axe unidimensionnel.

En second lieu, si l'on étend nos observations aux entités bi- ou tridimensionnelles ainsi qu'aux entités abstraites, il n'est pas non plus évident de vérifier la réalisation du postulat (ii), selon lequel il y aurait une relation d'équidistance entre $\mathrm{NP}_{0}$ et $\mathrm{NP}_{1}$ qui permettrait en 
particulier d'opposer au centre de et au milieu de. Dans le cas d'un $\mathrm{NP}_{1}$ abstrait, le calcul de l'équidistance est suspendu à cause de l'absence de forme géométrique :

(4) a. Au \{centre / ?milieu\} de \{tout / la totalité\}.

b. Au $\{$ ?centre / milieu $\}$ de rien.

Dans le même ordre d'idée, il est impossible de s'appuyer sur la forme géométrique de la poussière ou de la saleté pour expliquer le caractère peu naturel de 5 :

(5) Jean vit au \{?centre / milieu\} de la \{poussière / saleté\}.

10 Les exemples 6, quant à eux, montrent que les noms de couleur suivent naturellement la locution au milieu de, mais beaucoup plus difficilement au centre de. A nouveau, il semble difficile d'expliquer cela en termes d'équidistance :

(6) a. Sur le pont d'un navire qui était là tout seul au \{milieu / *centre\} du bleu immense, un groupe de jeunes hommes se tenait, le torse nu, au soleil levant.

b. Sur les prairies lointaines, où des points roux, semés çà et là au \{milieu / *centre\}

du vert uniforme, indiquaient des bœufs et des vaches, des troupeaux errants.

11 Dans le cas des $\mathrm{NP}_{1}$ concrets la difficulté demeure. Par exemple, lorsqu'elles précèdent terre, les deux locutions sont permises mais c'est le sens de terre qui se trouve affecté :

(7) a. Nous avons fait un voyage au $\left\{{ }^{*}\right.$ milieu / centre $\}$ de la Terre.

b. Au milieu de la terre grasse, humide et campagnarde...

c. On dirait que les monuments fleurissent au milieu d'une terre inhabitée.

Un syntagme comme au milieu de la terre ne permet pas de désigner de façon naturelle la planète du même nom, mais renvoie à la matière $(7 b)$ ou à l'étendue $(7 c)$. Loin d'être exceptionnel, on retrouve ce fonctionnement avec beaucoup d'autres compléments. Ainsi, lorsque monde suit la locution au milieu de, il prend un sens collectif et devient synonyme de "société » (exemple 8a). A l'inverse, la locution au centre de met en avant une autre acception de monde, synonyme de « cosmos » ou « univers » (exemples 8b-c) :

(8) a. En se sentant malheureuse au \{milieu / ?centre\} du monde et de ses fêtes désertes pour elle, la pauvre petite ne comprit plus rien à l'admiration qu'elle y excitait, ni à l'envie qu'elle inspirait.

b. L'homme a cru que la terre était au \{centre / ?milieu\} du monde.

c. Chaque homme a la folie de se croire au \{centre / ?milieu\} du monde.

13 Le nom de lieu générique espace illustre lui-même les limites d'une conception uniquement fondée sur les propriétés spatiales des compléments. Dans l'exemple $9 \mathrm{a}$, où il suit au milieu de, espace désigne le milieu extérieur à l'atmosphère terrestre. Mais après la locution au centre de il ne peut que renvoyer à un espace de stationnement, un espace fumeur, un espace de jeu ou encore un espace virtuel, un espace culturel, économique ou commercial :

(9) a. Au \{milieu /* centre $\}$ de l'espace, les radiations proviennent de toutes les directions.

b. Le dessin est situé au \{milieu / centre\} de l'espace de stationnement.

L'hypothèse que nous avons défendue dans (Gréa, 2005) est la suivante : la forme spatiale, géométrique, physique de $\mathrm{NP}_{1}$ ou sa dimension n'ont pas de valeur discriminante ; ce qui permet de distinguer au milieu de et au centre de tient essentiellement au caractère délimité ou non de $\mathrm{NP}_{1}$. A première vue, on pourrait penser que cette notion de délimitation découle assez naturellement des questions de forme géométrique et d'équidistance. Nous pensons au contraire qu'une différence fondamentale les oppose. La délimitation n'est pas ici à comprendre comme un concept géométrique. En particulier, elle n'implique pas la possibilité d'une bordure et d'un calcul d'équidistance. Il s'agit d'un concept plus général et plus abstrait. Pour le comprendre, revenons un instant à 
l'exemple 4. Le pronom tout ou le nom totalité se caractérisent, selon (Kleiber, 1998), par leur délimitation ${ }^{1}$. Or, ce n'est pas parce que tout a une délimitation que je peux pour autant dire que tout a une bordure, ou que je peux effectuer un calcul d'équidistance sur ce tout, ou que je peux lui donner une forme et une dimension particulière. Pourtant, c'est bien une délimitation de cet ordre que au centre de exige de la part de son complément, contrairement à au milieu de. Inversement, c'est l'absence d'une telle délimitation qui permet d'expliquer l'impossibilité d'un massif (poussière) ou d'un intensif (saleté) après au centre de. C'est encore une question de délimitation qui permet de distinguer les acceptions de terre (au sens délimité de planète, ou bien comme nom de matière non délimité), les acceptions de monde (au sens délimité d'univers, ou bien non délimité de société humaine) et les acceptions du terme espace qui exige, par l'adjonction d'un complément de nom, comme dans espace de stationnement, une clôture qu'il n'a pas de luimême, pour pouvoir être précédé de la locution au centre de.

Plusieurs arguments, que nous nous contenterons d'évoquer faute de place ${ }^{2}$, confirment l'importance de la délimitation comme critère distinctif. Le premier est le changement d'interprétation (distributif vs. collectif) que l'on observe avec les $\mathrm{NP}_{1}$ pluriels :

(10) a. Les conditions physiques qui règnent au centre des étoiles.

$\rightarrow$ dans les étoiles, au centre

$\rightarrow$ *parmi les étoiles

b. Les conditions physiques qui règnent au milieu des étoiles.

$\rightarrow$ ?dans les étoiles, au milieu

$\rightarrow$ parmi les étoiles

Dans l'exemple 10a l'interprétation la plus naturelle est celle où ce sont les conditions qui règnent au centre de chaque étoile qui sont terribles, c'est-à-dire une interprétation distributive. Ce point se trouve confirmé par le fait qu'il est toujours possible de restructurer le syntagme prépositionnel (les conditions physiques qui règnent dans les étoiles, au centre). En revanche, nous avons plutôt affaire à une interprétation collective dans $10 \mathrm{~b}$ et la zone localisée ne se trouve plus à l'intérieur des étoiles mais correspond à la portion d'espace qui se tient entre les étoiles ${ }^{3}$. Dans ce dernier cas, la restructuration du syntagme semble assez peu naturelle (?Les conditions physiques qui règnent dans les étoiles, au milieu).

On observe donc qu'avec un complément pluriel au centre de privilégie une lecture distributive alors que au milieu de privilégie une lecture collective. Or, cette observation valide notre hypothèse puisqu'en s'appliquant à chaque étoile, au centre de s'applique ainsi à des entités délimitées (en l'occurrence, de forme sphérique), et cela tout au contraire de au milieu de qui pointe sur l'espace situé entre les étoiles, c'est-à-dire un espace dénué de clôture. Ces observations n'impliquent pas que au centre des étoiles est systématiquement distributif. Cette expression peut prendre une interprétation collective, mais à la condition que le pluriel étoiles dénote un groupe. ${ }^{4} \mathrm{Ce}$ sera le cas, par exemple, si les étoiles en question sont celles d'une constellation ou d'un amas. Ce point confirme alors notre hypothèse, dans la mesure où un pluriel groupe présente une délimitation que n'a pas la simple pluralité.

On trouve un autre exemple de ce basculement collectif - distributif avec os. En effet, les expressions au milieu des os et au centre des os ne désignent pas la même zone, comme l'attestent les exemples suivants :

(11) a. Au \{centre / ?milieu\} des os, il y a la moelle osseuse.

b. Les os incisifs sont très larges et remontent jusqu'au \{milieu / centre\} des os du nez. 
19 Dans le premier cas, au centre des os s'applique exclusivement à chaque os pris individuellement. Cette lecture est moins fréquente avec au milieu qui a tendance à privilégier les interprétations collectives ou bien les affections pathologiques sur l'os (et non à l'intérieur : les fractures sont moins dangereuses au milieu des os). L'expression au centre des os peut prendre une interprétation collective. Pour cela, il faut que pluriel les os dénote un groupe : les os du nez (ou du crâne, etc.). C'est ce qu'illustre l'exemple $11 \mathrm{~b}$ où au centre des os s'applique au groupe d'os correspondant au nez.

Les $\mathrm{NP}_{1}$ massifs constituent un autre argument. Comme de nombreux auteurs l'ont remarqué ${ }^{5}$ les massifs se caractérisent par une absence de limites inhérentes :

«Pour notre propos, est fondamentale l'existence de $\mathrm{N}$ dénotant des substances présentées par la langue comme dépourvues de limites propres et qui, par là même, en reçoivent de l'extérieur ; car toute substance, continue ou non, se donne à nous à l'intérieur de limites: de même qu'on ne peut pas faire l'expérience de la totalité des êtres humains, de même on ne fait pas l'expérience de la totalité du vin. Le vin se présente, dans l'expérience empirique, enserré dans des limites, mais celles-ci ne lui sont pas inhérentes, à la différence de celles d'un être humain ou d'un vélo. » (Flaux et al., 2000, pp. 63-64)

21 Or, comme on le voit dans les exemples suivants, les massifs posent de grandes difficultés après au centre de, confirmant ainsi notre hypothèse selon laquelle la délimitation est une condition nécessaire pour cette locution :

(12) a. Il marche au \{milieu/*centre\} de la terre labourée sans relever le bas de son pantalon. (cf. ex. 7)

b. Voyage au centre de la terre.

(13) a. Les dernières vitres dégringolèrent, et au $\{$ milieu/*centre $\}$ du verre un âne attaché, qui ne tentait pas de fuir, se mit à braire désespérément sous la pluie qui recommençait à tomber.

b. Il faut verser la bière au centre du verre pour compléter le faux col.

(14) a. La toundra. Un pays sans arbres. Au \{milieu/*centre\} de la pierre, de la mousse et des cycles capricieux des caribous [...]

b. On aperçoit un défaut au centre de la pierre \{précieuse / tombale / à pizza\}.

(15) a. Ce n'est pas facile de travailler au \{milieu/*centre\} de la peinture.

b. On aperçoit un personnage grimaçant au centre de la peinture.

Le raisonnement est identique avec les pluriels amplifiants ${ }^{6}$ :

(16) a. Je préfère mourir de soif au \{milieu / *centre\} des sables que rester ici.

b. Le désert de Rome, aussi isolée sur la terre que Venise au \{milieu / *centre\} des eaux.

c. Ses porteurs se sont démoralisés et ont failli l'abandonner au \{milieu / ${ }^{*}$ centre\} des glaces.

d. On voit, au \{milieu / *entre\} des airs, quelque cigogne, attardée par la fatigue, qui se met à crier.

e. Je suis encore ici seul au \{milieu / * centre\} des neiges et de quatorze degrés de froid.

f. Puis, alentour, épars au \{milieu / *centre\} des terres, c'étaient les villages. ou les noms intensifs ${ }^{7}$ :

(17) a. Tout à coup, au \{milieu / *centre\} du désordre, au \{milieu / ${ }^{*}$ centre\} de l'effarement, au \{milieu / *centre\} de l'hostilité de la foule, ...

b. Au \{milieu / *entre\} du chagrin, la concupiscence me torture.

c. Au \{milieu / *centre\} de la haine exaspérée des uns, de l'indignation vraie ou feinte des gens du monde, du mépris envieux de quelques confrères, du respect de toute une foule de lecteurs, et de l'admiration frénétique d' un grand nombre.

d. Son accès se termina dans un désir sentimental d'une vie simple, le cœur sur la 
main, au \{milieu / *centre\} d'une bonté universelle.

e. Au \{milieu / *centre\} d'un ennui indéfinissable. / milieu de. Notre objectif, en effet, est de confronter des concepts que les noms centre et milieu ne mettent pas directement en œuvre, à savoir l'opposition cible / site telle qu'elle est classiquement présentée dans le cadre des grammaires cognitives, et l'opposition figure / fond telle qu'elle est caractérisée dans la théorie de la Gestalt. Néanmoins, comme nous aurons l'occasion de l'évoquer (section 3.2), nous pensons que les conclusions auxquelles nous arrivons à propos des locutions demeurent valables pour les noms.

\section{La signification fonctionnelle de $\mathrm{NP}_{0}$}

\subsection{Au centre de : une relation partie-tout?}

Un premier exemple montre que le critère de délimitation de $\mathrm{NP}_{1}$ n'est pas suffisant :

(18) a. Ribéry évolue au centre du trio d'attaque.

b. Ribéry évolue au milieu du trio d'attaque.

Dans 18a, la première interprétation qui vient à l'esprit est celle selon laquelle Ribéry fait partie du trio d'attaque. Dans 18b, au contraire, Ribéry n'appartient pas nécessairement au trio. Or, une chose est sûre, ce changement d'interprétation (partitive vs. non partitive) n'est pas lié à la délimitation de $\mathrm{NP}_{1}$.

Il faut donc explorer une autre piste. Dans la section précédente, nous nous sommes intéressé au cas du pronom tout et du nom totalité (ex. 4). A cette occasion, nous avons évoqué le rapport que Kleiber établit entre la notion de totalité et l'existence de limite : 
«On peut accepter en première approche que tout, parce qu'il indique la totalité, nécessite d'une part un domaine de quantification borné et d'autre part une structure interne partitive de ce domaine. La première exigence s'explique aisément: je ne puis parler de totalité que pour une entité qui a des limites. L'absence de frontières nous prive en effet du moyen de calculer la totalité. La seconde n'est qu'une conséquence définitoire de la notion de totalité : celle-ci n'existe que si l'on pose en même temps l'existence de parties, donc d'une division possible de l'objet en parties ${ }^{10}$. ( (Kleiber, 1998, p 90) convaincant dans la mesure où il marque une opposition nette entre au centre de et au milieu de. L'explication fondée sur une relation partie-tout est donc séduisante. Il faut pourtant y renoncer en raison de sa trop grande puissance. Comme le montrent les exemples suivants, la relation partie-tout est loin d'être systématique :

(20) a. La soupière était au centre de la table ${ }^{*} \rightarrow$ La soupière était une partie de la table.

b. Un grand arbre était au centre des tables $* \rightarrow$ Un grand arbre faisait partie des tables

c. La table, au centre du trio, déborde de victuailles* $\rightarrow$ La table fait partie du trio.

Les exemples 18, 19 et 20 montrent toutefois que même si la relation partie-tout est trop forte, elle n'est pas sans rapports avec le problème qui nous occupe. Les sections suivantes vont être l'occasion de montrer pourquoi.

\subsection{La localisation sur le corps}

33 Un certain nombre d'exemples appartenant au domaine concret permettent d'avancer une autre hypothèse. Il s'agit de la localisation sur le corps, comme c'est le cas, par exemple, avec la relation vêtement-partie du corps. On peut localiser les vêtements par rapport à la partie du corps qu'ils sont censés recouvrir. Mais dans ce cas de figure, seul au milieu de est possible, tandis que au centre de est exclu :

(21) a. La casquette au \{milieu / *entre\} du front.

b. Le tee-shirt, trop petit, lui arrive au \{milieu / *centre\} du ventre.

c. Une chemise dont les manches arrivent au \{milieu /*centre\} des bras. 
d. Le slip lui descend au \{milieu / *entre\} des fesses.

e. Le col lui arrive au \{milieu / *entre\} du menton.$$
\begin{aligned}
& \text { L'idée que nous défendrons est fondée sur la signification fonctionnelle de } \mathrm{NP}_{0} \text { : la moelle } \\
& \text { entretient en effet une relation fonctionnelle intrinsèque avec l'os, tandis que le tee-shirt }
\end{aligned}
$$
se tient dans un simple rapport de superposition avec le ventre.

\subsection{La question des noms collectifs}

40 Un second cas de figure permet d'illustrer cette idée en opérant une autre distinction entre au milieu de et au centre de. Il s'agit d'une classe de noms d'extension spatiale exprimant une pluralité et une structuration interne importante, tels que système, dispositif, échiquier, réseau, toile, organisation. Selon la locution choisie, la localisation de NP 
${ }_{0}$ sur $\mathrm{NP}_{1}$ ne connait pas de changement fondamental mais on observe en revanche un type de rapport entre $\mathrm{NP}_{0}$ et $\mathrm{NP}_{1}$ très différent. Considérons les exemples suivants :

(24) a. La Chine est au centre de l'échiquier asiatique.

b. Il faut replacer le citoyen au centre de l'organisation politique.

c. L'étoile est au centre du système solaire.

d. Il y avait au centre de ces formations politiques, des états-majors dont les

objectifs n'étaient pas du tout inspirés par une volonté émanée de la base.

Dans ces exemples, le sens de la locution au centre de ne correspond pas seulement à une localisation de $\mathrm{NP}_{0}$ par rapport à $\mathrm{NP}_{1}$. Il s'agit aussi d'indiquer que $\mathrm{NP}_{0}$ a un rôle fonctionnel par rapport à $\mathrm{NP}_{1}$. Du coup, la double condition - à savoir l'obligation pour NP ${ }_{1}$ de correspondre à une entité délimitée et pour $\mathrm{NP}_{0}$ d'entretenir un rapport fonctionnel avec NP1 - favorise nettement la lecture partie-tout (de manière comparable à l'exemple 18a) : dans ces exemples, la Chine fait partie de l'échiquier politique, le citoyen fait partie de l'organisation politique, l'étoile fait partie du système solaire et les états-majors font partie des formations politiques. A l'inverse, si la locution au milieu de permet une localisation qu'on pourrait croire " comparable $»^{14}$, elle ne confère aucune signification fonctionnelle à $\mathrm{NP}_{0}$ :

(25) a. ?La Chine est au milieu de l'échiquier asiatique.

b. ?Le président est au milieu de l'organisation politique.

c. ?L'étoile est au milieu du système solaire.

d. ?Il y avait au milieu de ces formations politiques, des états-majors dont les

objectifs n'étaient pas du tout inspirés par une volonté émanée de la base.

Du coup, l'impression qui ressort de ces exemples, au-delà de leur caractère peu naturel, est que la Chine, le citoyen, l'étoile et les états-majors sont certes localisés, mais qu'aucune relation fonctionnelle n'est établie avec le $\mathrm{NP}_{1}$. La conséquence est la difficulté d'obtenir une lecture partitive dans ces contextes. On a au contraire le sentiment que la Chine, le citoyen, l'étoile et les états-majors sont comme « déconnectés » de $\mathrm{NP}_{1}$ et qu'ils sont là «par hasard ».

\subsection{Les noms d'extension temporelle}

A l'exception des noms intensifs abordés dans les exemples 17 , tous les $\mathrm{NP}_{1}$ étudiés jusqu'ici ont une extension spatiale. Or, comme nous allons le voir dans ce qui suit, notre hypothèse, qui s'appuie sur le rôle fonctionnel de $\mathrm{NP}_{0}$ par rapport à $\mathrm{NP}_{1}$, trouve aussi une confirmation avec la classe des noms de processus, et plus généralement, avec tous les noms ayant une extension temporelle.

Les emplois temporels sont à l'origine d'une divergence très claire entre les deux locutions. Comme on le voit dans les exemples suivants, la locution au milieu de prend automatiquement une valeur temporelle lorsqu'elle précède un nom de mesure temporelle :

(26) a. La révolte a éclaté au \{milieu / *centre\} \{de la journée / de la semaine / du mois / de l'année\}.

b. C'était au milieu du jour. (Nerval)

Ce n'est pas le cas de au centre de qui est impossible dans ce contexte, à moins de prendre en compte le genre littéraire :

(27) Au centre du jour, jeté dans le tas des sardines voyageuses d'un coléoptère à

l'abdomen blanchâtre, un poulet au grand cou déplumé... 
auquel sont associés des rôles thématiques (cf. section 2-5). Or, ces observations semblent contredire l'explication avancée dans notre introduction, à savoir la délimitation de $\mathrm{NP}_{1}$ lorsqu'il est sous la portée de au centre de. En effet, dans les exemples et un film ou une semaine correspondent à des étendues temporelles qui ont un début et une fin; ce sont des entités bornées. Par conséquent, la locution au centre de, qui exige un complément délimité, devrait pouvoir apparaître dans ce contexte tout en conservant un sens temporel. Or, ce n'est pas le cas et l'énoncé *Pierre part au centre du film est inacceptable.

5 suffisante. Les sections précédentes ont en effet montré l'existence d'une seconde contrainte permettant d'opposer les deux locutions : la signification fonctionnelle de $\mathrm{NP}_{0}$ par rapport au tout dénoté par $\mathrm{NP}_{1}$. Or, les exemples 26 et 28 ne respectent pas cette seconde contrainte. Ainsi, le déclenchement de la révolte ou le départ de l'étudiant n'entretiennent aucune relation fonctionnelle intrinsèque avec l'étendue temporelle dénotée par semaine, cours ou film. Pour mieux comprendre ce point, il est utile de faire le lien avec les contextes vestimentaires des exemples 21 , car de la même manière que le rapport entre un vêtement et une partie du corps est un simple rapport de superposition, le départ de l'étudiant, dans l'exemple 28, entretient avec cours ou film un simple rapport de concomitance. Dans les deux cas, la relation entre $\mathrm{NP}_{0}$ et $\mathrm{NP}_{1}$ est une relation de coïncidence qui implique une absence de rapport fonctionnel intrinsèque entre les deux arguments, et qui amène ainsi à privilégier la locution au milieu de au détriment de au centre de. Il en est tout autrement des exemples 29. Dans ces contextes, le rapport entre $\mathrm{NP}_{0}$ et $\mathrm{NP}_{1}$ n'est pas une coïncidence mais correspond à un rapport fonctionnel intrinsèque, celui qui unit de façon essentielle un évènement et son thème. Dans $29 a$ et $29 \mathrm{~b}$, en effet, $\mathrm{NP}_{0}$ (la généricité ou la violence en milieu rural) est ce sur quoi porte $\mathrm{NP}_{1}$ (respectivement, la semaine de recherche ou le film de Pierre) : $\mathrm{NP}_{0}$ est le thème de $\mathrm{NP}_{1}$. Il est donc doté d'une signification fonctionnelle qui fait toute la différence avec les emplois purement temporels. 


\subsection{Au centre de : une alternance de valence inédite}

52 On le voit, au centre de est susceptible d'introduire des noms de processus, mais à la condition que ces derniers ne soient pas pris dans leur acception temporelle. A partir d'un corpus constitué à l'aide de Glossanet ${ }^{16}$, nous avons établi l'index (classé par ordre de fréquence décroissante et non lemmatisé) de ces noms prédicatifs qui entrent dans la construction $\left[\mathrm{NP}_{0}\right.$ au centre de $\left.\mathrm{NP}_{1}\right]$ et qui en viennent ainsi à entretenir un rapport fonctionnel avec $\mathrm{NP}_{1}$ :

Tableau : Index de fréquence des $\mathrm{N} 1$ dans $\left[\mathrm{NP}_{0}\right.$ au centre de $\left.\mathrm{NP}_{1}\right]$ (Corpus : Glossanet)

\begin{tabular}{|c|c|c|}
\hline Rang & N1 & Exemples \\
\hline 1 & entretiens & Les pressions israélo-américaines au centre des entretiens. \\
\hline 2 & débats & Une tentative de meurtre sera au centre des débats les 22 et 23 janvier. \\
\hline 3 & discussions & Le terrorisme et le gaz au centre des discussions algéro-espagnoles. \\
\hline 4 & campagne & l'intercommunalité n'a pas été au centre de la campagne électorale. \\
\hline 5 & polémique & Accident mortel: Les responsabilités au centre de la polémique. \\
\hline 6 & débat & Le concept de souveraineté est au centre du débat politique libanais. \\
\hline 7 & scandale & Le groupe Sanlu, au centre du scandale du lait contaminé par la mélamine. \\
\hline 8 & réunion & La question a été au centre de la réunion de mercredi. \\
\hline 9 & intérêts & Le droit au retour des réfugiés au centre des intérêts libanais. \\
\hline 10 & revendications & L'annulation de la dette est au centre des revendications. \\
\hline 11 & affaire & Ce produit est au centre d'une affaire récente. \\
\hline 12 & controverse & Au centre de la controverse, une loi votée hier. \\
\hline 13 & discussion & Le port du voile était au centre de la discussion. \\
\hline 14 & discours & La crise régionale a été au centre des discours prononcés par $M . *$ \\
\hline 15 & interrogations & Equipe de France de foot: le milieu au centre des interrogations. \\
\hline 16 & priorités & Les préoccupations environnementales au centre des priorités. \\
\hline 17 & référendum & Le Kosovo au centre du référendum sur la Constitution. \\
\hline 18 & rencontre & Ce projet de réformes devait être au centre de la rencontre. \\
\hline 19 & trafic & Le soigneur de l'équipe est au centre d'un trafic de produits dopants. \\
\hline
\end{tabular}




\begin{tabular}{|c|c|c|}
\hline 20 & attaques & $\begin{array}{l}\text { Au centre des attaques: le journaliste Andrew Gilligan, qui avait discrètement } \\
\text { interviewé David Kelly, scientifique au ministère de la Défense. Se référant à } \\
\text { cette source anonyme, le reporter avait affirmé sur les ondes de la } B B C \text { que } \\
\text { Londres avait exagéré la menace des armes de destruction massive de Saddam } \\
\text { Hussein avant la guerre. }\end{array}$ \\
\hline 21 & attentions & $\begin{array}{l}\text { Le mouvement des joueurs dans le football est au centre des attentions chaque } \\
\text { année }\end{array}$ \\
\hline 22 & conflit & Les deux enfants (12 et 6 ans) sont au centre du conflit, bien malgré eux. \\
\hline 23 & attention & $\begin{array}{l}\text { Ce phénomène [le recul de la banquise] est au centre d'une attention } \\
\text { considérable, tant de la part des scientifiques que des industries extractrices ou } \\
\text { des Etats. }\end{array}$ \\
\hline 24 & conversation & Au centre de la conversation, bien sûr, le nucléaire iranien. \\
\hline 25 & conversations & Le conflit au Proche-Orient sera au centre des conversations. \\
\hline 26 & exposition & $\begin{array}{l}\text { Ce ne sont pas les cuuves de Dali, mais le peintre lui-même dans son acte de } \\
\text { création qui est au centre de l'exposition. }\end{array}$ \\
\hline 27 & incident & $\begin{array}{l}\text { Le joueur s'est fait remarquer lors de ce tournoi en étant au centre d'un incident } \\
\text { qui a valu à l'Angleterre d'être éliminé. }\end{array}$ \\
\hline 28 & inquiétudes & L'attentat remet le royaume wahhabite au centre des inquiétudes. \\
\hline 29 & négociations & Le pétrole justement. Il est au centre des négociations. \\
\hline 30 & réflexion & Cette évolution est au centre de la réflexion du philosophe. \\
\hline 31 & voyage & L'immigration chinoise en France, au centre du voyage officiel en Chine. \\
\hline
\end{tabular}

Dans tous ces exemples, il est à noter que la locution au milieu de est exclue. Pour qu'elle redevienne naturelle, il faudrait imposer une lecture temporelle portant sur l'intervalle de temps occupé par l'évènement dénoté par le nom prédicatif (par exemple : il est parti au milieu de l'entretien). Mais dans ce cas de figure, la relation entre $\mathrm{NP}_{0}$ et $\mathrm{NP}_{1}$ reste une simple coïncidence et le fait de partir n'a pas de rapport fonctionnel avec l'entretien. Il en est tout autrement de la locution au centre de. Elle a pour effet de mettre en relief la structure argumentale de $\mathrm{NP}_{1}$, et ce, de trois façons différentes. Comme nous allons le montrer maintenant, au centre de est en effet à l'origine d'une alternance de valence inédite (ci-dessous, i et ii) et d'un cas d'ambigüité tout aussi remarquable (iii).

(i) Le premier cas de figure correspond aux exemples 29 et s'avère être le plus fréquent. Dans ce contexte, la construction $\left[\mathrm{NP}_{0}\right.$ au centre de $\left.\mathrm{NP}_{1}\right]$ se glose de la façon suivante: " $\mathrm{NP}_{1}$ porte sur / a pour thème $\mathrm{NP}_{0}$ ", ce qu'illustrent les exemples suivants :

(30) a. Les pressions israélo-américaines au centre des entretiens. ( $\rightarrow$ les entretiens portent / ont pour thème les pressions israélo-américaines).

b. Une tentative de meurtre sera au centre des débats les 22 et 23 janvier.

c. Le terrorisme et le gaz au centre des discussions algéro-espagnoles.

d. L'intercommunalité n'a pas été au centre de la campagne électorale. 

fonctionnelle évidente par rapport à $\mathrm{NP}_{1}$, signification fonctionnelle qui se trouve suspendue lorsqu'on substitue au milieu de à au centre de :

(31) ?Les pressions israélo-américaines au milieu des entretiens.

Le changement de sens est alors considérable, et s'il faut réinterpréter l'énoncé, il semble que le sens temporel, selon lequel des pressions ont eu lieu ou ont été évoquées au cours des entretiens, soit le plus naturel.

(ii) Le second cas de figure, plus rare, a pour effet d'inverser le schéma argumental précédent. Dans ce contexte, la construction $\left[\mathrm{NP}_{0}\right.$ au centre de $\left.\mathrm{NP}_{1}\right]$ se glose de la façon suivante: " $\mathrm{NP}_{0}$ est la cause / à l'origine de $\mathrm{NP}_{1}$ ", comme l'illustrent les exemples suivants :

(32) a. Le soigneur de l'équipe est au centre d'un trafic de produits dopants $\rightarrow$ Le

soigneur est la cause / le responsable / à l'origine du trafic)

b. Ragnar Rylander a bien été au centre d'une fraude scientifique. ${ }^{17}$

Par exemple, dans 32a, on comprend que le soigneur est à l'origine du trafic, et non que le trafic a pour thème le soigneur. En d'autres termes, la signification fonctionnelle de $\mathrm{NP}_{0}$ par rapport à $\mathrm{NP}_{1}$ est bien avérée, mais elle est d'un tout autre genre que précédemment : $\mathrm{NP}_{0}$ est cette fois l'instigateur de l'action dénotée par $\mathrm{NP}_{1}$. Et comme dans la situation précédente, cette valeur fonctionnelle disparaît lorsqu'on commute avec au milieu de :

(33) Le soigneur de l'équipe est au milieu d'un trafic de produits dopants. $\mathrm{NP}_{0}$ et $\mathrm{NP}_{1}$ devient un simple rapport de coïncidence qui s'exprimera plus naturellement avec se retrouver (le soigneur se retrouve au milieu d'un trafic).

(iii) Un troisième cas de figure correspond à une ambiguïté entre (i) et (ii). C'est le cas des exemples suivants :

(34) a. Le prince Charles au centre du complot.

b. Bourse : La situation de la Lybie au centre des inquiétudes.

c. Assises du Hainaut : l'argent au centre d'un crime crapuleux.

61 Sans contexte supplémentaire, il est impossible de décider si le prince Charles est l'instigateur du complot ou bien la victime. Mais ce qui importe, c'est que dans les deux cas, le prince Charles est doté d'une signification fonctionnelle par rapport au complot. Comme prévu, cette signification fonctionnelle disparaît avec la locution au milieu de :

(35) Le prince Charles au milieu du complot.

Ici, en effet, Charles n'est plus impliqué dans le complot (que ce soit comme instigateur ou comme cible) et le rapport entre $\mathrm{NP}_{0}$ et $\mathrm{NP}_{1}$ revient alors à un simple rapport de coïncidence, ce qui, du reste, n'est pas sans poser quelques problèmes quant à l'interprétation exacte de l'énoncé. ${ }^{18}$

Les contraintes imposées par au centre de semblent suffisamment systématiques pour permettre de tester la polysémie des déverbaux, entre acception spatiale, temporelle, évènementielle, et dans ce dernier cas, de tester la structure argumentale du déverbal (instigateur / thème). Pour illustrer ce point, prenons le cas des noms assistance, assemblée et réunion. Les deux premiers ont une acception spatiale que l'on peut vérifier à l'aide des deux locutions. Ce n'est pas le cas de réunion qui paraît moins naturel dans ce contexte :

(36) Le ministre se trouvait au \{milieu / centre\} de \{l'assistance /de l'assemblée /

*de la réunion\}. 
64

anche, seuls les deux derniers (assemblée et réunion) ont une acception temporelle que la locution au milieu de permet de tester en portant cette fois sur l'étendue temporelle occupée par l'évènement :

(37) Il a décidé de partir au milieu de \{*l'assistance / de l'assemblée / de la réunion\}. Dans ces contextes, au milieu de a un rôle équivalent à pendant.

Assemblée et réunion sont enfin susceptibles de dénoter un évènement. Au centre de permet alors de tester les rôles d'instigateur et/ou de thème :

(38) La laïcité est au centre de \{*l'assistance / la réunion / ?l'assemblée\}.

On observe ainsi que assistance, bien que déverbal de assister, ne passe pas dans ce contexte : assistance n'a ni thème ni instigateur, contrairement à réunion. Assemblée est plus naturel dans ce contexte s'il s'agit explicitement d'une assemblée organisée par quelqu'un en vue de quelque chose :

(39) a. Les préoccupations matérielles au centre de l'assemblée générale.

b. Le sujet est au centre de l'assemblée plénière ordinaire des évêques.

c. La référence au communisme au centre de l'assemblée extraordinaire du PCF.

d. Religion : La laïcité au centre de l'assemblée des évêques.

Le cas des noms intérêt, ennui et mépris illustre encore les différences que la locution au centre de est à même d'établir parmi les déverbaux. Les verbes correspondants (respectivement, s'intéresser, s'ennuyer et mépriser) semblent avoir une structure argumentale comparable, comme le montrent les deux exemples suivants :

(40) a. La situation sociale grecque intéresse le ministre.

b. La situation sociale grecque ennuie le ministre.

c. Le ministre méprise la situation sociale grecque.

Or, les déverbaux ne se comportent pas de la même façon sous la portée de au centre de :

(41) a. La situation sociale grecque est au centre de l'intérêt du ministre.

b. *La situation sociale grecque est au centre de l'ennui du ministre.

c. *La situation sociale grecque est au centre du mépris du ministre.

L'explication de cette divergence est peut-être à rechercher dans la valeur agentive associée au nom prédicatif intérêt - valeur que l'on retrouve dans tous les $\mathrm{N}_{1}$ du Tableau 1 - et l'absence d'agentivité dans le cas de ennui et mépris. ${ }^{19}$

\section{Qu'est ce que la localisation?}

\subsection{Le géométral comme condition de la localisation}

Lorsqu'on choisit de travailler dans un cadre référentialiste, les notions de distance, d'équidistance, et plus généralement, de métrique, sont les notions clés permettant de caractériser le sens de centre et milieu : le centre est ce qui est à équidistance des bords. A première vue, un tel constat relève du bon sens. Prenons l'exemple d'un avion. Il respecte la contrainte de délimitation appliquée à $\mathrm{NP}_{1}$. L'empennage entretient en outre avec l'ensemble un rapport fonctionnel intrinsèque puisqu'il garantit la stabilité en vol. La seconde condition, celle d'une signification fonctionnelle de $\mathrm{NP}_{0}$, est donc elle aussi vérifiée. Or, il serait très étrange, et manifestement faux, de dire :

(42) L'empennage est au centre de l'avion.

puisque l'empennage est... à l'arrière de l'avion. C'est bien la preuve que au centre de a un sens fondamentalement lié, d'une manière ou d'une autre, à une question d'équidistance. La suite d'un tel raisonnement nous amène alors à donner la priorité à des questions de 
formes géométriques, de dimension, de topologie. On en arrive ainsi à penser, par exemple, qu'une locution comme au centre de doit avoir un site au moins bidimensionnel. Dans cette optique, on en vient à classer comme inacceptable des énoncés tel que au centre de la ligne.

71 Derrière la force et la simplicité de ce raisonnement, il y a l'idée que toute analyse sémantique de la localisation doit reposer sur la possibilité d'une représentation objective de la chose, de sa mesure géométrique réelle. Pour le dire dans les termes de la phénoménologie de Merleau Ponty, l'analyse référentialiste de la localisation est entièrement soumise à l'ordre d'un "géométral », à savoir, dans le cas présent, une géométrie "naturelle", c'est-à-dire non formalisée et directement accessible à l'intuition, par rapport à laquelle les différentes perspectives seraient unifiées et coordonnées entre elles. ${ }^{20}$ La notion d'équidistance, par exemple, participe entièrement d'un tel géométral, en donnant à croire qu'il existe une métrique objective grâce à laquelle il serait possible de reconstituer, derrière les différentes perspectives, la forme vraie de l'objet, et à partir de laquelle on pourrait, inversement, dériver toutes les perspectives possibles. Il en est de même des considérations fondées sur la dimension de $\mathrm{NP}_{1}$ (uni-bi-tridimensionnel) ou sur sa forme (plutôt circulaire dans le cas de centre, et plutôt imprécise dans celui de milieu). Dans cette approche, les locutions au milieu de et au centre de ne sont rien d'autre que deux façons distinctes de localiser les choses par rapport à cette réalité commune. C'est très précisément ce genre de point de vue qui amène de nombreux chercheurs à minimiser la différence, par exemple, entre les syntagmes suivants :

(43) a. au milieu de la table

b. au centre de la table

Ces deux syntagmes sont considérés comme synonymes parce qu'on croit disposer d'un géométral qui les coordonne, parce qu'on pense qu'ils se rapportent à une seule et même réalité objective.

Notre but n'est pas de remettre en cause ce point de vue, mais d'inverser l'ordre des priorités. Au lieu de construire notre raisonnement sur la base de l'existence d'un géométral, nous proposons de partir du point de vue inverse, selon lequel ce qui est localisé par les deux locutions ne correspond pas, même dans les cas d'apparente synonymie. Cela est évident lorsqu'on compare, par exemple, les syntagmes au milieu de la terre et au centre de la terre: dans le premier cas un élément autonome se trouve superposé sur un fond qui n'a pas de limite spécifiée (cf. ex. $7 \mathrm{~b}-\mathrm{c}$ ), tandis que dans le second, il y a un rapport de signification fonctionnelle avec un tout délimité (ex. 7a). Ces deux perspectives sont très différentes l'une de l'autre : elles ne se rapportent pas à une réalité commune et donnent lieu à des contextes qui n'ont aucun rapport. A propos de tels exemples, il devient difficile de soutenir l'existence d'une géométrie naturelle qui serait cachée derrière les deux perspectives: les situations dénotées par centre et milieu sont incommensurables. Dans ces conditions, on soutiendra qu'il en est de même avec au milieu de la table et au centre de la table.

La dernière section sera donc l'occasion de renoncer à tout géométral, à toute métrique commune, à tout repère susceptible de coordonner le milieu et le centre, et de voir quelle caractérisation sémantique devient possible dans ces conditions. 


\subsection{Pour une approche phénoménologique et gestaltiste de la localisation} exactement cette opposition. Intéressons-nous d'abord au cas de la construction $\left[\mathrm{NP}_{0}\right.$ au milieu de $\mathrm{NP}_{1}$ ]. Les sections précédentes ont permis de montrer que cette locution constitue ${ }^{22} \mathrm{NP}_{0}$ en élément autonome qui n'entretient pas d'autre rapport avec $\mathrm{NP}_{1}$ que celui d'une simple superposition. Par ailleurs, $\mathrm{NP}_{1}$ est lui-même constitué en une entité dont la délimitation n'est pas spécifiée. Cette locution permet donc d'établir un rapport entre $\mathrm{NP}_{0}$ et $\mathrm{NP}_{1}$ qui rassemble toutes les propriétés d'un rapport figure - fond tel qu'il est conçu par la théorie de la gestalt. Dans ce cadre, en effet, une figure est définie comme une entité délimitée superposée sur un fond non délimité avec lequel il n'entretient aucune relation fonctionnelle. Dans ces conditions, les pluriels (ex. 10b), les massifs (ex. 12a, 13a, 14a et 15a), les pluriels amplifiants (ex. 16) et les intensifs (17) prennent naturellement ce rôle de fond, dans la mesure où, comme nous l'avons dit, ils n'ont pas de délimitation spécifiée (cf. section 1). Dans le cas où $\mathrm{NP}_{1}$ dénote un processus temporel délimité, la construction $\left[\mathrm{NP}_{0}\right.$ au milieu de $\left.\mathrm{NP}_{1}\right]$ permet de marquer la simple concomitance de deux évènements (ex. 26 et 28).

77

. lui-même constitué en un tout délimité. Dans ces conditions, $\mathrm{NP}_{1}$ n'a pas le statut d'un fond, mais celui d'une figure. En cela, $\mathrm{NP}_{1}$ est délimité et peut correspondre, dans les faits, aux atomes d'une pluralité (ex. 10a et 11a), à un pluriel ayant une interprétation de type groupe (ex. 11b), à un comptable (ex. 12b, 13b, 14b et 15b), ou encore à un évènement ayant un instigateur ou un thème intrinsèque (ex. 29, 30, 32 et 34). L'existence d'une relation fonctionnelle entre $\mathrm{NP}_{0}$ et $\mathrm{NP}_{1}$ a une conséquence importante : $\mathrm{NP}_{0}$ n'est pas un élément conçu comme isolé, indépendant de $\mathrm{NP}_{1}$. Au contraire, si la construction $\left[\mathrm{NP}_{0}\right.$ au centre de $\mathrm{NP}_{1}$ ] n'implique pas nécessairement une relation partie-tout (ex. 20), elle est susceptible de l'exprimer chaque fois que le contexte s'y prête (ex. 18 et 19) et devient automatique lorsque $\mathrm{NP}_{1}$ désigne une pluralité (ex. 10a et 11a, à moins que la pluralité ne corresponde à un pluriel groupe, comme dans l'ex. 11b). Dans tous ces cas de figure, à 
cause de son rôle fonctionnel, $\mathrm{NP}_{0}$ est constitué par au centre de comme étant, pour ainsi dire, « partie prenante » de $\mathrm{NP}_{1}$.

Cette analyse a de multiples conséquences que nous n'avons pas la place de développer. L'une d'entre elles mérite toutefois d'être soulignée. En imposant une relation fonctionnelle à un tout délimité, au centre de favorise des objets ayant une structuration interne hétérogène. A l'inverse, au milieu de, en imposant un rapport de superposition non fonctionnel sur un fond, tend à privilégier des objets ayant une structuration interne homogène. Ce sont des propriétés qui sont reconduites dans la sémantique des noms noyaux. Les pluriels les milieux et les centres, comme le remarque (Honeste, 2007), illustrent parfaitement ce point : le milieu politique (sing.) et le les milieux politiques (pl.) sont susceptibles de renvoyer au même objet du fait de l'homogénéité interne de milieu. Il en est tout autrement du couple le centre politique vs. les centres politiques où la structuration interne hétérogène de centre fait de lui un comptable délimité et donne lieu à une véritable pluralisation. ${ }^{23}$

Quoiqu'il en soit, les deux locutions donnent accès à deux perspectives très différentes. Ce n'est pas comme si ces dernières se rapportaient à un même objet sur lequel il s'agirait de localiser des zones plus ou moins comparables. Il faut au contraire renverser la perspective habituelle: les considérations géométriques de forme et de distance - qui servent de géométral aux analyses référentielles - n'occupent plus le centre du dispositif, mais se trouvent reléguées à leur véritable rôle, celui de principes subalternes uniquement applicable à un cas de figure particulier, celui des emplois spatiaux mettant en jeu un $\mathrm{NP}_{0}$ comptable ( au \{milieu / centre\} de l'avion, cf. ex. 42). Et même dans ce cas, la non convergence du centre et du milieu est toujours susceptible de reprendre le dessus. C'est ce que montrent les exemples 3 , où au centre de la table en vient finalement à désigner l'un des convives. Or, on en conviendra facilement, ce dernier se trouve doté d'une signification fonctionnelle particulière qui n'est qu'assez secondairement reliée au fait qu'il se trouve à équidistance des limites de la tablée.

Parmi les conséquences de ce renversement de situation, il en est une qu'il faut mettre en avant. Comme nous l'avons vu, la locution décide du statut de ses compléments : au milieu de impose le statut de fond à $\mathrm{NP}_{1}$, tandis que au centre de lui impose celui de figure. Or, cette distribution ne coïncide pas avec l'opposition cible - site que l'on utilise classiquement dans le traitement de la localisation spatiale. ${ }^{24}$ Pour les deux locutions, $\mathrm{NP}_{1}$ correspond au site (repère) et $\mathrm{NP}_{0}$ à la cible (entité localisée). L'opposition figure - fond que nous venons de mettre au jour relève donc d'un tout autre niveau d'organisation.

\section{BIBLIOGRAPHIE}

Borillo, A., 1998. L'espace et son expression en français. Paris : Ophrys.

Borillo, A., 1999. « Partition et localisation spatiale : les noms de localisation interne », Langages 136, 53-75.

Flaux, N., 1999. « A propos des noms collectifs », Revue de linguistique romane 63, 471-502. 
Flaux, N. \& Van de Velde, D., 2000. Les noms en français : esquisse de classement. Paris / Gap : Ophrys.

Gréa, P., 2005. «Les locutions au \{centre / milieu\} de dans leur acception spatiale », Revue de Sémantique et de Pragmatique 18, 87-112.

Gréa, P. 2012, « 'Deux-trois’ mots sur la question des déterminants de petite quantité : pluriel continu et perception sémantique ». Journal of French Language Studies. Disponible à : CJO doi : 10.1017/S0959269512000191

Guillaume, P., 1937. La psychologie de la forme. Paris : Flammarion.

Gurwitsch, A., 1957. Théorie du champ de la conscience. Paris : Desclée de Brouwer.

Honeste, M.-L., 1997. « La notion d'espace en français à travers l'étude de quelques mots de très haute fréquence ", in C. Rivière \& M.-L. Groussier (eds), La Notion. Paris : Ophrys. 290-296.

Honeste, M.-L., 1997. « De la dénomination aux stratégies argumentatives : l'exemple d'espace et centre », in Cl. Boisson \& Ph. Thoiron (eds), Autour de la dénomination. Lyon : Publications de l'Université de Lyon, 279-305.

Honeste, M.-L., 2007. « Lexique français de la spatialisation et représentations mentales », in P. Bacot \& S. Rémi-Giraud (eds), Mots de l'espace et conflictualité sociale. Paris : L'Harmattan, collection « Langue et parole », 79-88.

Kleiber, G., 1997. « Massif / comptable et partie / tout », Verbum 3, 321-327.

Kleiber, G., 1998. « Tout et ses domaines : sur la structure tout + déterminant + N », in A. Englebert, M. Pierrard, L. Rosier \& D. van Raemdonck (eds), La ligne claire : de la linguistique à la grammaire, Mélanges offerts à Marc Wilmet à l'occasion de son $60^{\text {ème }}$ anniversaire. Louvain / Paris : Duculot, 87-97.

Köhler, W., 1929 [1964]. Psychologie de la forme. Paris : Gallimard.

Langacker, R.W., 1987. Foundations of Cognitive Grammar: Theoretical Prerequisites. vol. 1. Stanford: Stanford University Press.

Langacker, R.W., 1991a. Foundations of Cognitive Grammar: Descriptive Application. vol. 2. Stanford: Stanford University Press.

Langacker, R.W., 1991b. « Noms et verbes », Communications 53, 103-154.

Langacker, R.W., 2008. Cognitive Grammar. A Basic Introduction. Oxford: Oxford University Press. Link, G., 1983. « The Logical Analysis of Plurals and Mass Terms: A Lattice-Theoretical Approach ", in R. Bäuerle, C. Schwarze \& A. von Stechow (eds), Meaning, Use, and Interpretation of Language. Berlin: Walter de Gruyter.

Maingueneau, D., 1993. Eléments de linguistique pour le texte littéraire. Paris : Nathan.

Merleau-Ponty, M., 1945. Phénoménologie de la perception. Paris : Gallimard.

Renouard, M., 2001. « Le point de vue de Sirius et la cartographie du visible », in J. Benoist \& F. Merlini (eds), Historicité et spatialité. Le problème de l'espace dans la pensée contemporaine. Paris : Vrin.

Schnedecker, C., 2008. « Tout nom et tout pronom : qu'est ce qui fait la différence ? », in J. Durand, B. Habert \& B. Laks (eds), Congrès Mondial de Linguistique Française - CMLF'08. Paris : 2031-2050. 
Talmy, L., 1978. « Figure and Ground in Complex Sentences », in J. Greenberg (ed), Universals of Human Language 4. Stanford : Stanford University Press, 625-649.

Vandeloise, C., 1986. L'espace en français. Paris : Seuil.

Van de Velde, D., 1995. Le spectre nominal. Des noms de matières aux noms d'abstraction. Louvain / Paris : Peeters.

Vieu, L., 1991. Sémantique des relations spatiales et inférences spatio-temporelles : une contribution à l'étude des structures formelles de l'espace en Langage Naturel. Thèse de doctorat. IRIT, Université Paul Sabatier.

Wertheimer, M., 1923. « Untersuchungen zur Lehre von der Gestalt, II », Psychologische Forschung 4, 301-350.

\section{NOTES}

1. Comme le souligne (Kleiber, 1998) : "Je ne puis parler de totalité que pour une entité qui a des limites ». A noter toutefois que (Schnedecker, 2008) rediscute en partie cette analyse.

2. Mais que nous développons dans (Gréa, 2005).

3. Et c'est le cas pour la trentaine d'occurrences de au milieu des étoiles dans Frantext.

4. Cf. (Link, 1983).

5. (Langacker, 1991b : 117 et suiv.) (Kleiber, 1997) (Flaux et al., 2000) entre autres.

6. Cf. (Maingueneau, 1993).

7. Cf. (Van de Velde, 1995). Si l'absence de délimitation est commune aux massifs et aux intensifs, les massifs ont une étendue dans l'espace, alors que les intensifs n'ont d'étendue ni spatiale ni temporelle.

8. "Chaque monade est un point de vue sur le monde, qu'elle exprime exactement comme une projection de perspective exprime le plan géométral à partir duquel elle est tracée ; c'est-à-dire qu'il y a entre ce qui peut se dire de l'une et de l'autre un rapport constant et réglé » (Renouard, $2001: 203)$

9. «l'un après l'autre et selon l'apparence perceptive, je ne construis pas l'idée du géométral qui rend raison de ces perspectives mais le cube est déjà là devant [nous] et se dévoile à travers eux » (Merleau-Ponty, 1945 : 237).

10. A noter que le caractère purement analytique (par définition) de ce dernier argument n'est pas si évident puisqu'il entre en contradiction avec le constat, par le même auteur (Kleiber, 1997), qu'il y a des comptables sans structuration interne. Par ailleurs, on pourrait aussi discuter du statut a priori du premier argument, selon lequel la notion de totalité implique nécessairement celle de limite: il se pourrait qu'il ne soit pas tout à fait valide dans le domaine des mathématiques, par exemple, en fonction de la définition que l'on donne à la notion de limite ou de borne.

11. Le fait que seule une partie du tee-shirt soit concernée par la localisation - à savoir, le bas du tee-shirt, tandis que le reste couvre les épaules - ne semble pas entrer en ligne de compte : il suffit de se reporter à l'attache de l'exemple $22 \mathrm{~b}$ pour constater que le problème est identique lorsque $\mathrm{N}_{0}$ est entièrement contenu dans les limites de $\mathrm{N}_{1}$.

12. Explication suggérée par B. Fagard (com. pers.).

13. Sur ce point, on peut aussi renvoyer aux exemples 12-15 dans lesquels au centre de sélectionne systématiquement le sens comptable, c'est-à-dire délimité, par opposition à au milieu de, qui ne pose aucun problème avec le sens massif, c'est-à-dire non délimité. On peut aussi se reporter à la citation de Flaux et al (2000) supra que nous prenons comme base de travail.

14. Cf. néanmoins la section 3 pour l'incommensurabilité des deux types d'énoncés. 
15. Comme pour les énoncés spatiaux, il ne s'agit pas d'un calcul (B. Fagard, com. pers.).

16. Constitué à partir d'une veille de plusieurs mois sur GlossaNet, à partir d'une vingtaine de journaux francophones : http://glossa.fltr.ucl.ac.be.

17. Ragnar Rylander est un chercheur suédois qui a travaillé à l'Université de Genève tout en étant financé parallèlement par Philip Morris. Ce professeur était sous contrat avec le cigarettier depuis 1972 et il a dirigé des symposiums et produit des recherches minimisant ou niant les effets néfastes du tabac et du tabagisme passif. (source : wikipedia)

18. Problème d'interprétation que l'on retrouve dans les exemples 31,33 , mais aussi, et pour les mêmes raisons, dans les exemples 25.

19. Cet exemple est inspiré d'une observation que font (Flaux et al., 2000, p.31) à propos de intérêt et ennui (cf. aussi le tableau figurant p. 39).

20. La remarque est aussi valable pour le traitement que nous avons-nous-même proposé dans le cadre de la grammaire cognitive (cf. Gréa 2005, pp. 104 et suiv.).

21. Cf. (Gurwitsch, 1957 : 121-126). On peut renvoyer à (Gréa, 2012) pour une autre illustration de cette idée, dans le cadre d'une application de la Gestalttheorie à la pluralité (pluriel continu vs. discontinu).

22. Et non " profile", comme ce serait le cas si nous nous placions dans le cadre de la grammaire cognitive de Langacker. Le verbe profiler fait implicitement référence, sous ses déformations diverses, à la nécessaire constance de l'entité profilée, à savoir la base conceptuelle. Cette dernière joue alors le rôle de géométral et nous ramène ainsi dans le cadre général des analyses référentialistes.

23. Cf. (Kleiber, 1997).

24. Cf. (Talmy, 1978) (Vandeloise, 1986) (Langacker, 1987 et 1991a).

\section{RÉSUMÉS}

Les noms centre et milieu sont des Noms de Localisation Interne (NLI) pouvant se combiner avec la préposition $\grave{a}$, et construire de la sorte des locutions prépositionnelles locatives $\mathrm{NP}_{0}$ au milieu de $N P_{1}$ et $N P_{0}$ au centre de $N P_{1}$. Dans un cadre référentialiste, leur caractérisation s'appuie sur une représentation géométrique du sens de centre et de milieu (forme spatiale et dimension de $\mathrm{NP}_{1}$ ). L'objectif de cet article est de remettre en question ce point de vue en montrant que la distinction entre au milieu de et au centre de tient dans deux paramètres sémantiques: (i) le caractère délimité ou non de $\mathrm{NP}_{1}$ et (ii) la signification fonctionnelle de $\mathrm{NP}_{0}$ par rapport à $\mathrm{NP}_{1}$. $\mathrm{A}$ l'aide de ces deux critères nous rendrons compte de données qui vont du domaine concret (noms concrets singuliers et pluriels, massifs, collectifs) au domaine abstrait (noms intensifs, noms d'action).

L'étude de ces deux locutions est en outre un bon prétexte pour proposer une approche de la localisation qui s'inscrit dans un arrière-plan philosophique inhabituel, celui de la phénoménologie. Nous développerons l'idée que les effets de perspectives induits par les locutions au centre / milieu de ne se rapportent pas à une réalité commune, et que ce qui est localisé par une locution ne coïncide jamais avec ce qui est localisé par l'autre, même dans les cas d'apparente synonymie. Pour cela, nous reprendrons l'opposition gestaltiste figure / fond, et nous montrerons que au centre de établit une relation fonctionnelle avec un tout délimité (une 
forme), tandis que au milieu de établit un rapport de coïncidence avec un fond dont la délimitation n'est pas spécifiée.

Centre (center) and milieu (middle) are Nouns of Internal Localization (NIL). They combine with the preposition à to form complex locative preposition phrases such as $N P_{0}$ au milieu de $N P_{1}$ (at the middle of) and $N P_{0}$ au centre de $N P_{1}$ (at the center of). Referentialist semantics characterizes these constructions with reference to a geometric interpretation of centre and milieu. This paper challenges the referentialist approach and shows that the distinction between au milieu de and au centre de hinges on two semantic criteria. The first criterion has to do with whether $\mathrm{NP}_{1}$ is bounded or not. The second criterion concerns the functional meaning of $\mathrm{NP}_{0}$ with respect to $\mathrm{NP}_{1}$ - These two criteria account for linguistic phenomena that range from the concrete domain (concrete nouns in the singular and in the plural, mass nouns, collective nouns) to the abstract domain (intensifying names and action names).

The study of these two preposition phrases is also a good opportunity to propose a renewed approach to localization based on philosophy, and more precisely phenomenology. We develop the following idea : au centre de/au milieu de impose distinct perspectivizing effects and localize radically different elements, even when these elements are apparently synonyms. To substantiate these claims, we use the Gestalt-based opposition between figure and ground. We show that au centre de makes a functional link with a bounded whole (a figure), whereas au milieu de implies a coincidence with a substance whose boundaries are not specified.

INDEX

Keywords : collective interpretation, distributive interpretation, theme, instigator, geometral, figure / ground

Mots-clés : interprétation collective, interprétation distributive, thème, instigateur, géométral, figure / fond

\section{AUTEUR}

\section{PHILIPPE GRÉA}

MoDyCo - Université Paris 10 \& CNRS 